\title{
NOTE ON THE DEFINING RELATIONS FOR THE SIMPLE GROUP OF ORDER 660
}

F. A. LEWIS

Dickson* has given the following set of defining relations for the simple group of order 660 :

$$
\begin{array}{cc}
S^{11}=I, & T^{2}=I, \quad(S T)^{3}=I, \\
& \left(S^{3} T S^{8} T\right)^{2}=I, \\
& \left(S^{4} T S^{6} T\right)^{2}=I .
\end{array}
$$

It follows from (3) that

$$
T S^{4} T=S^{5} T S^{7} T S^{5},
$$

so that

$$
\begin{aligned}
T S^{8} T & =S^{5} T S^{7} T S^{10} T S^{7} T S^{5} \\
& =S^{5} T S^{8} \cdot S^{-1} T S^{-1} T S^{-1} \cdot S^{8} T S^{5} \\
& =S^{5} T S^{8} T S^{8} T S^{5} .
\end{aligned}
$$

From this last relation

$$
\begin{aligned}
S^{3} T S^{5} T S^{5} \cdot S^{3} T S^{8} T \cdot S^{5} T & =I, \\
S^{3} T S^{8} T & =S^{6} T S^{6} T S^{8} T S^{6}, \\
\left(S^{3} T S^{8} T\right)^{2} & =S^{6} T S^{6} T S^{8} T S T S^{6} T S^{8} T S^{6} \\
& =S^{6} T S^{6} T S^{7} \cdot S T S T S \cdot S^{5} T S^{8} T S^{6} \\
& =S^{6} T S^{6} \cdot T S^{7} T S^{5} T \cdot S^{8} T S^{6} \\
& =S^{6} T S^{6} \cdot S^{6} T S^{4} \cdot S^{8} T S^{6} \\
& =S^{6} T S T S T S^{6} \\
& =I .
\end{aligned}
$$

Since (2) has been derived from (1) and (3), it follows that (1) and (3) are sufficient to define the simple group of order 660.

\section{University of Alabama}

\footnotetext{
* This Bulletin, vol. 9 (1903), pp. 204-206.
} 